\title{
Phase noise and jitter reduction by optical feedback on passively mode-locked quantum-dot lasers
}

Cite as: Appl. Phys. Lett. 103, 231101 (2013); https://doi.org/10.1063/1.4837716

Submitted: 25 July 2013 • Accepted: 13 November 2013 • Published Online: 02 December 2013

\section{Arsenijević, M. Kleinert and D. Bimberg}
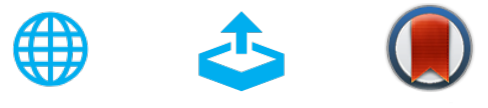

\section{ARTICLES YOU MAY BE INTERESTED IN}

rf linewidth reduction in a quantum dot passively mode-locked laser subject to external optical feedback

Applied Physics Letters 96, 051118 (2010); https://doi.org/10.1063/1.3299714

Low noise performance of passively mode locked quantum-dash-based lasers under external optical feedback

Applied Physics Letters 95, 131111 (2009); https://doi.org/10.1063/1.3238324

Perspective: The future of quantum dot photonic integrated circuits

APL Photonics 3, 030901 (2018); https://doi.org/10.1063/1.5021345

耳QBLOX

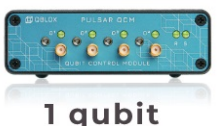

Shorten Setup Time Auto-Calibration More Qubits

Fully-integrated Quantum Control Stacks Ultrastable DC to $18.5 \mathrm{GHz}$ Synchronized $<<1$ ns Ultralow noise

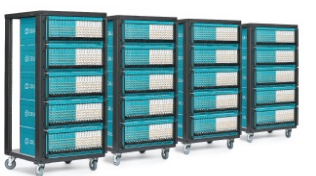

100s qubits

visit our website > 


\title{
Phase noise and jitter reduction by optical feedback on passively mode-locked quantum-dot lasers
}

\author{
D. Arsenijević, ${ }^{\text {a) }}$ M. Kleinert, ${ }^{\text {b) }}$ and D. Bimberg ${ }^{\text {() }}$ \\ Institut für Festkörperphysik, EW 5-2, Technische Universität Berlin, Hardenbergstraße 36, \\ 10623 Berlin, Germany
}

(Received 25 July 2013; accepted 13 November 2013; published online 2 December 2013)

\begin{abstract}
The noise properties of the pulse trains of passively mode-locked $40 \mathrm{GHz}$ quantum-dot lasers subject to optical feedback are investigated in detail. Five different feedback regimes are discovered and the clearly identified regime of resonant optical feedback is further examined. The feedback parameters yielding minimum phase noise are determined. Here, the radio-frequency (RF)-line-width is reduced from its original value by $99 \%$ to $1.9 \mathrm{kHz}$. The corresponding pulse-to pulse jitter of $23 \mathrm{fs}$ is a record low value for passively mode-locked $40 \mathrm{GHz}$ quantum-dot lasers. (C) 2013 AIP Publishing LLC. [http://dx.doi.org/10.1063/1.4837716]
\end{abstract}

Optical pulse sources with repetition rates of several tens of Gigahertz are essential for today's optical data communication networks. Because of their small footprint, simplicity, and low production costs, monolithic quantum-dot (QD) mode-locked lasers (MLLs) are ideal devices for such applications since they can be fabricated in monolithically integrated way. ${ }^{1}$ Large noise figures however are strongly restricting their applications. Hybrid mode locking is a possible solution reducing the residual phase noise and timing jitter of the emitted pulse train. Indeed, very low timing jitter has been demonstrated, but the required stable highfrequency electrical oscillator represents a large cost disadvantage. ${ }^{2}$ Injection of narrow-bandwidth laser light (e.g., from an external cavity laser) to a $10 \mathrm{GHz}$ MLL was shown to reduce the radio-frequency ( $\mathrm{RF}$ )-line-width from several hundreds of kilohertz down to below $1 \mathrm{kHz}$ and to reduce the noise. ${ }^{3}$ This approach suffers from the same disadvantage as hybrid mode-locking of high costs caused by the external light source. Recently, optical feedback (OFB), similar to what has been demonstrated several decades ago for directly modulated lasers, ${ }^{4,5}$ has been revisited as a simple and effective way to reduce the phase noise of MLL-pulse-trains. ${ }^{6,7} \mathrm{~A}$ reduction of the RF-line-width in the electrical spectrum from $46 \mathrm{kHz}$ to $1.1 \mathrm{kHz}$ is reported for a $5.25 \mathrm{GHz}$ QD MLL. ${ }^{8}$ For a $10 \mathrm{GHz}$ single-section InP-based quantum-dash laser, a reduction of the 3 -dB-line-width from $30 \mathrm{kHz}$ to $200 \mathrm{~Hz}$ was shown by Akrout et al. ${ }^{9}$ However, OFB was also reported to degrade the pulse properties of MLLs. ${ }^{10}$ In this paper, we investigate the impact of three crucial parameter fields of OFB on two-section passively MLLs in detail. Five fundamentally different regimes including the decisive one leading to enormous improvements of the pulse properties are discovered. The results we present are not restricted to the particular wavelength of the laser used here but are of a general nature. Inexpensive low noise multi-ten $\mathrm{GHz}$ laser modules can now find their way into systems.

\footnotetext{
a)dejan@sol.physik.tu-berlin.de

b) Present address: Fraunhofer Heinrich Hertz Institute (HHI), Einsteinufer 37, 10587 Berlin, Germany.

${ }^{c}$ Also at King Abdul Aziz University, Jeddah, Saudi Arabia.
}

The devices investigated in this paper are grown by molecular beam epitaxy on an n+-doped GaAs substrate. The emission wavelength is close to $1310 \mathrm{~nm}$. 10 layers of InAs QDs in a dot-in-a-well structure are stacked. ${ }^{11} \mathrm{~A}$ device length of around $1 \mathrm{~mm}$ ascertains a fundamental modelocking frequency of $38.616 \mathrm{GHz}$, close to $40 \mathrm{GHz}$. Absorber and gain sections of the MLL are electrically separated by a trench using chemically assisted ion beam etching. The saturable absorber has a length of $110 \mu \mathrm{m}$ and its facet has a high reflection coating (99\%). The gain section facet is as cleaved. The device is mounted on a copper block and the temperature is controlled by a thermo-electric cooler which was set to $21^{\circ} \mathrm{C}$. For all measurements, the gain current was $68 \mathrm{~mA}$ and the absorber bias was $-5 \mathrm{~V}$. This corresponds to the optimal operation point of such MLLs at which stable mode locking occurs. The average optical output power in fiber is $2.8 \mathrm{~mW}$.

The OFB is realized by injecting a part of the laser light back into the cavity through the facet at the gain section. Our single-mode-fiber-based feedback loop comprises an optical circulator, a 9:1-splitter, a variable attenuator, an optical delay line, and a polarization controller as shown in Fig. 1. $10 \%$ of the emitted intensity can thus be used for the OFB. This fiber-based approach enables to adjust the three essential parameters of the OFB easily. A coarse adjustment of the round-trip time of the pulses inside the feedback loop can be accomplished by varying the length of the optical fiber. A fine adjustment is done by tuning the optical delay line (delay between 0 ps and $200 \mathrm{ps)}$ ). The feedback strength is controlled by the variable attenuator. The overall attenuation inside the feedback loop with respect to the laser output power is tuned between $16 \mathrm{~dB}$ and $52 \mathrm{~dB}$. The laser emission is analyzed using an autocorrelator as well as an electrical spectrum analyzer in combination with a fast photo diode.

From the electrical spectra, the RF peak power $P(f)$ and the RF-frequency $f$ are extracted. Furthermore, the integrated timing jitter $\sigma^{R M S}$ as a measure of the phase noise is calculated from single side-band phase noise traces ${ }^{12}$

$$
\sigma^{R M S}=\frac{1}{2 \pi f} \sqrt{2 \int d(\Delta f) P_{\text {Noise }}(\Delta f)}
$$




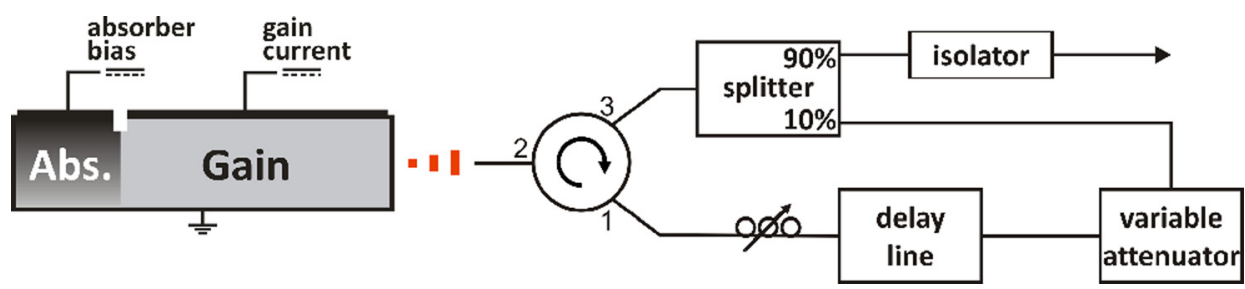

FIG. 1. Functional plot of the setup.

where $P_{\text {Noise }}$ denotes the phase noise power density at the frequency offset $\Delta f$ (with respect to the carrier frequency). In this investigation, the integrated timing jitter is calculated using integration borders of $10 \mathrm{kHz}$ and $1 \mathrm{GHz}$, respectively. By varying the above mentioned feedback parameters and observing the changes in RF-frequency, peak power, and integrated timing jitter, as well as in the autocorrelation of the pulses, different feedback regimes are identified. Figure 2(a) shows the peak power of the RF-spectrum as a function of delay and feedback attenuation. The delay interval of 27.5 ps is sufficient because it has previously been shown that the effects of OFB are periodic with a period given by the pulse distance (here $25 \mathrm{ps}$ ). ${ }^{6}$ Five different OFB-regimes can be identified in Fig. 2(a). Figure 2(b) shows representative single sideband phase noise traces for each regime. These five regimes are surprisingly similar to the feedback regimes reported for distributed feedback lasers. ${ }^{5}$ Regime 1 is located at feedback attenuations smaller than $20 \mathrm{~dB}$. It is characterized by low peak power of the RF-spectrum (not shown here). Up to noise frequencies of $5 \mathrm{MHz}$, the phase noise is similar to the case without OFB. Therefore, the width of the central line in the RF-spectrum remains constant. In addition, strong sidebands in the RF-spectrum occur across a frequency range of $1.5 \mathrm{GHz}$. They correspond to the peaks in the phase noise trace at frequencies of multiples of $12.4 \mathrm{MHz}$. This frequency depends on the round-trip time inside the feedback loop. Furthermore, the autocorrelation trace of the pulses in regime 1 shows a strong cw-background: In regime 1, mode locking is deteriorated by OFB. In regime 2, the MLL exhibits chaotic behavior with changes of the RF-spectrum within a couple of seconds. Phase noise traces could not be measured. In regime 3 , the side-bands in the RF-spectrum are smaller than in regime 1 but the width of the central line is enhanced yielding a larger phase noise at low frequencies. The cw-background in the autocorrelation traces is not as large as in regime 1 , but larger than without OFB: in the regime 3, mode locking is deteriorated by the OFB as well. Regime 4 is the case of resonant optical feedback. The peak power in the RF-spectrum rises by $10 \mathrm{~dB}$. The corner frequency of the white noise plateau in the phase noise trace is shifted from $80 \mathrm{kHz}$ to 10 $\mathrm{KHz}$. The thermal noise level is reached at a noise frequency of $1 \mathrm{MHz}$. The side-bands with a separation of $12.4 \mathrm{MHz}$ are $30 \mathrm{~dB}$ weaker than in regime 1 and 3 . Thus, the singlesideband noise is strongly suppressed compared to the free running case. At the same time, the autocorrelation of the pulses is not influenced in regime 4. Details of this regime will be explained in the next paragraph. In regime 5, only small influences of the OFB can be observed. The autocorrelation of the pulses is unaffected, but the line width in the RFspectrum fluctuates between the one without OFB and larger widths. Therefore, the phase noise can increase in regime 5. This is the reason why MLLs are usually operated in conjunction with optical isolators to prevent instabilities of the pulse emission. For practical purposes, it is obviously desirable to operate a MLL in the regime 4 of the OFB. In what follows, we will focus on the resonant $\mathrm{OFB}$ in that regime.

It has been shown that in the case of resonant feedback an integer number $q$ of pulses propagate through the feedback loop. ${ }^{6,7}$ The round-trip time inside the feedback loop $\tau_{F B}$ for a pulse distance $\tau_{M L L}=f^{-1}$ in the resonant feedback case is

$$
\tau_{F B}=q \tau_{M L L}
$$

By changing the feedback length, e.g., via the delay, the MLL repetition rate changes in order to meet this criterion. Figure 3 shows the repetition rate of the MLL at an attenuation of $34 \mathrm{~dB}$ for different feedback regimes (compare Figure 2(a)). In the regime 4 , the repetition rate exhibits a clear dependence on the delay, which agrees with the prediction for resonant feedback. The repetition rate is pulled by the change in the delay. In the regimes 3 and 5, such dependence is not observed. In both cases, the OFB is out of resonance. Regimes 3 and 5 therefore differ fundamentally from regime 4. The shape of regime 4 in Figure 2(a) can be explained by the pulling of the repetition rate under resonant feedback. At feedback attenuations larger than $45 \mathrm{~dB}$, the influence of the OFB is too small for such a pulling to occur. Stronger OFB
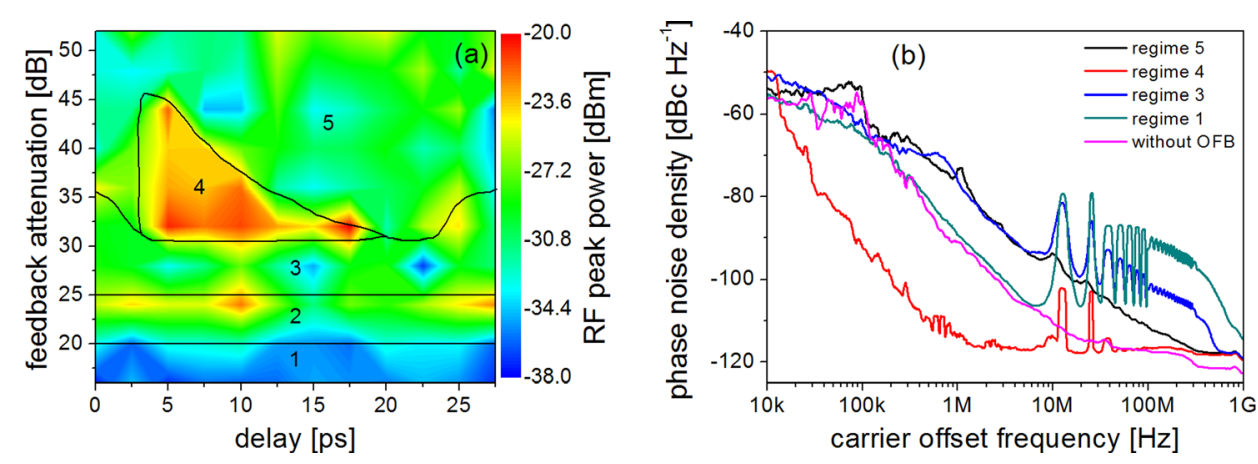

FIG. 2. A color coded map of the peak power as a function of delay and feedback attenuation (a).The single sideband phase noise traces in different OFB-regimes (b). For both figures, the feedback length is $16.6 \mathrm{~m}$. 


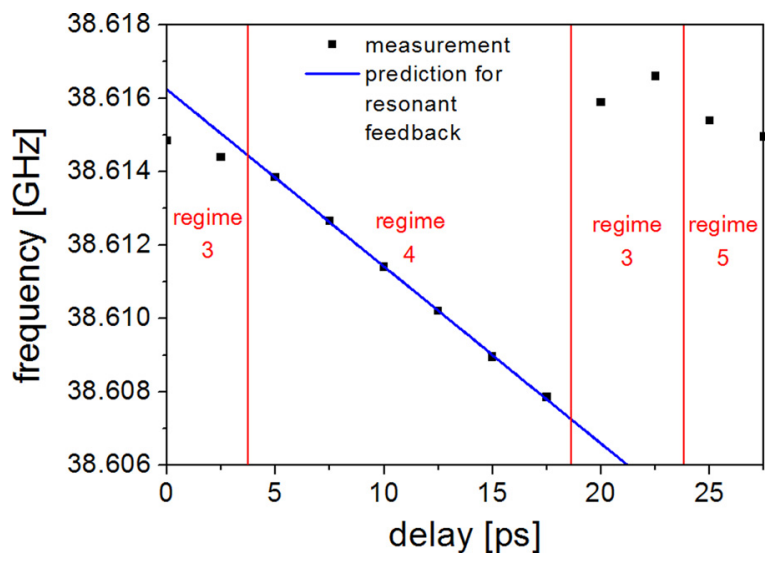

FIG. 3. The repetition rate of the MLL as a function of the delay at a feedback length of $16.6 \mathrm{~m}$ and a feedback attenuation of $34 \mathrm{~dB}$.

leads to the onset of the pulling at a delay of $3.5 \mathrm{ps}$. At this delay, the difference between the repetition rate of the MLL without OFB $(38.616 \mathrm{GHz})$ and the repetition rate induced by the resonant feedback is small. With larger delays, the difference between these two repetition rates increases. With stronger OFB, the repetition rate can be pulled further, extending the range of possible delays for resonant feedback. The largest extent of the regime 4 is reached at a feedback attenuation of $30 \mathrm{~dB}$ just before regime 3 sets in.

In order to maximize the extent of the resonant feedback regime, the fiber length inside the feedback loop is varied between $16.6 \mathrm{~m}$ up to $120 \mathrm{~m}$. An optimal fiber length of $31.7 \mathrm{~m}$ is found, at which the lowest jitter and the maximum extent of the resonant feedback are observed. At a fiber length of $31.7 \mathrm{~m}$, the regime 3 of the OFB vanishes and the regime 4 extents to a feedback attenuation of $27 \mathrm{~dB}$ and boarders directly with regime 2 . At feedback attenuations between $27 \mathrm{~dB}$ and $31 \mathrm{~dB}$, the resonant feedback regime is not interrupted by tuning the delay. The repetition rate of the MLL is pulled effectively and resonant feedback is obtained for all delay values. Figure 4 shows furthermore that the integrated jitter is reduced from 3.8 ps without OFB to values lower than 1 ps. Above attenuations of $31 \mathrm{~dB}$, the delay dependence of the regime 4 resembles the one known from Figure 2(a) (note the periodicity of $25 \mathrm{ps}$ ). At the border between the regimes 4 and 5 , the jitter can increase to more than 7 ps (white area). The independence of the resonant feedback regime on the delay at low attenuations is very favorable for practical applications: fluctuations of the feedback length do not cause switching into other OFB-regimes.

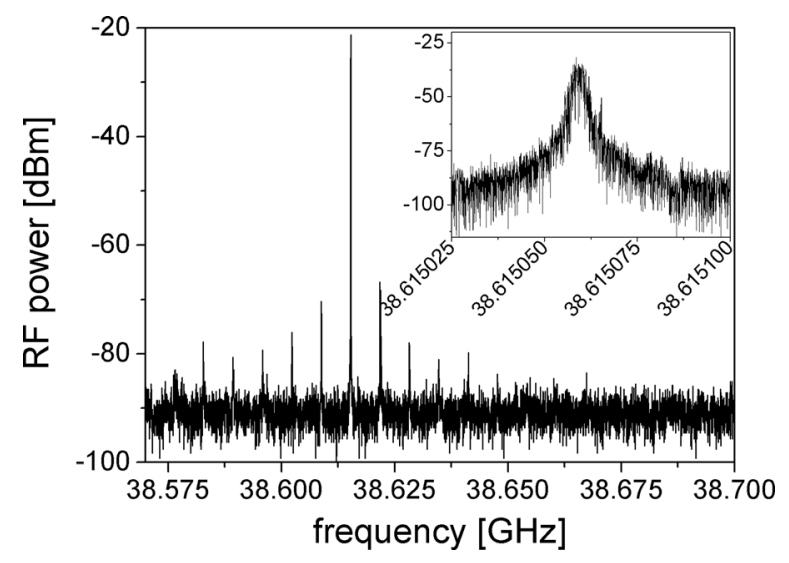

FIG. 5. The RF-spectrum of the MLL subject to optimal optical feedback. The inset shows the central line of the RF-spectrum.

The parameters for optimal OFB for the present MLL are a feedback length of $31.7 \mathrm{~m}$, a feedback attenuation of $29 \mathrm{~dB}$, and a delay of 19 ps. For these values, the central line in the RF-spectrum is very strong with a signal-to-noise ratio of $66 \mathrm{~dB}$. The sidebands are suppressed by at least $45 \mathrm{~dB}$. Their distance to the central line is given by multiples of 6.5 MHz, corresponding to the feedback length of $31.7 \mathrm{~m}$. The inset of Figure 5(b) shows the central line. The 3-dBline-width $\nu$ is reduced to $1.9 \mathrm{kHz}$. Compared to the line width of $187 \mathrm{kHz}$ without OFB, this presents a reduction by $99 \%$. The pulse-to-pulse jitter can be calculated as ${ }^{13}$

$$
\sigma_{P-P}=\sqrt{\frac{\nu}{2 \pi f^{3}}} .
$$

The pulse-to-pulse jitter and the integrated timing jitter are reduced from 228 fs to 23 fs and from 3.8 ps to 219 fs, respectively. These jitter and line width values are the lowest ever reported for passively mode-locked quantum-dot lasers. The integrated jitter for hybrid mode-locking is measured by us to be $250 \mathrm{fs}$. Optical feedback therefore presents an alternative to hybrid mode-locking having the huge advantage of simplicity and low cost.

The influence of optical feedback on passively MLLs is investigated in detail and five different regimes are identified. Theses regimes are characterized with respect to the stability of the pulse emission. The resonant feedback regime is of practical interest and offers a cost-effective way to stabilize the pulse emission of passively mode-locked lasers. We are now able to maximize the extent of this regime and to
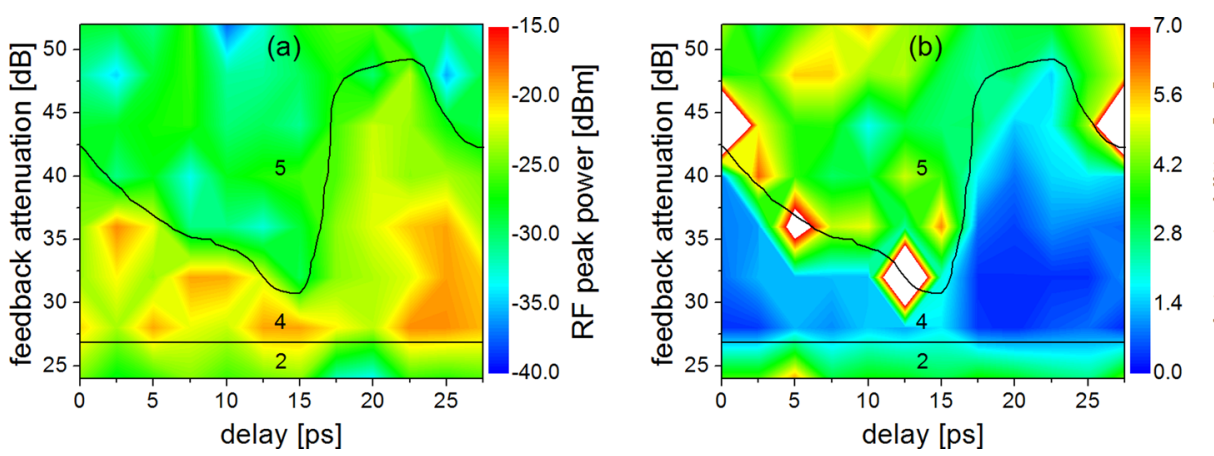

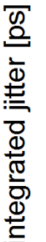

FIG. 4. A color coded map of the RF peak power (a) and the integrated jitter (b) as a function of delay and feedback attenuation at a feedback length of $31.7 \mathrm{~m}$. White areas correspond to values larger than $7 \mathrm{ps}$. 
determine the parameters for optimal optical feedback. At this point, strong suppression of the phase noise is observed leading to record low values for jitter and line width.

The authors would like to thank C. Otto for helpful theoretical discussions. The research leading to these results has received funding from the DFG in the framework of the SFB 787.

${ }^{1}$ D. Bimberg, J. Phys. D 38, 2055 (2005).

${ }^{2}$ M. Kuntz, G. Fiol, M. Laemmlin, C. Meuer, and D. Bimberg, Proc. IEEE 95, 1767 (2007).

${ }^{3}$ T. Habruseva, N. Rebrova, S. P. Hegarty, and G. Huyet, Lecture Notes Nanoscale Sci. Technol. 13, 65 (2012).

${ }^{4}$ K. Ketterer, E. H. Boettcher, and D. Bimberg, Appl. Phys. Lett. 50, 1471 (1987).
${ }^{5}$ R. W. Tkach and R. W. Chraplyvy, J. Lightwave Technol. 4, 1655 (1986).

${ }^{6}$ G. Fiol, M. Kleinert, D. Arsenijević, and D. Bimberg, Semicond. Sci. Technol. 26, 014006 (2011).

${ }^{7}$ C. Otto, K. Lüdge, A. G. Vladimirov, M. Wolfrum, and E. Schöll, New. J. Phys. 14, 113033 (2012).

${ }^{8}$ C.-Y. Lin, F. Grillot, Y. Li, R. Raghunathan, and L. F. Lester, IEEE J. Sel. Top. Quantum Electron. 17, 1311 (2011).

${ }^{9}$ A. Akrout, A. Shen, A. Enard, G.-H. Duan, F. Lelarge, and A. Ramdane, Electron. Lett. 46, 73 (2010).

${ }^{10}$ E. A. Avrutin, X. Song, and B. M. Russel, Opt. Quantum Electron. 40, 1175 (2008)

${ }^{11}$ A. R. Kovsh, N. A. Maleev, A. E. Zhukov, S. S. Mikhrin, A. P. Vasilev, E. A. Semenova, Yu. M. Shernyakov, M. V. Maximov, D. A. Livshits, V. M. Ustinov, N. N. Ledentsov, D. Bimberg, and Zh. I. Alferov, J. Cryst. Growth 251, 729 (2003).

${ }^{12}$ D. von der Linde, Appl. Phys. B 39, 201 (1986).

${ }^{13}$ F. Kefelian, S. O'Donoghue, M. T. Todaro, J. G. McInerney, and G. Huyet, IEEE Photon. Technol. Lett. 20, 1405 (2008). 